\title{
Use of 157-nm Photodissociation to Probe Structures of $y$ - and $b$-Type Ions Produced in Collision-Induced Dissociation of Peptide Ions
}

\author{
Liangyi Zhang and James P. Reilly \\ Department of Chemistry, Indiana University, Bloomington, Indiana, USA
}

$y$ - and $b$-type fragment ions produced in the collisional dissociation of arginine-terminated peptide ions are photodissociated with 157-nm light in a linear trap. $y$-type ions are shown to have the same structure as that of intact peptides of the same sequence with the ionizing proton located at the most basic residue(s). For generic $b$-type ions, the ionizing proton is shown to be sequestered at the N-terminal arginine, which is consistent with the proposed oxazolone structure. (J Am Soc Mass Spectrom 2008, 19, 695-702) (C 2008 American Society for Mass Spectrometry

$\mathrm{L}$ ow-energy collision-induced dissociation (CID) of singly protonated peptides produces primarily $y$ - and $b$-type ions and is generally well described by the mobile proton model, in which an ionizing proton moves to backbone amides and facilitates backbone cleavage [1-4]. Although formation of $b$-type ions obviously requires that the ionizing proton moves to the $\mathrm{N}$-terminal fragment and formation of $y$-type ions requires that it goes to the C-terminal fragment, the structures for these fragment ions are still under investigation.

It is generally accepted that the composition of $y$-type ions is that of truncated peptides of the same sequence. However, the position of the ionizing proton remains unclear. Biemann and colleagues originally proposed that the ionizing proton of $y$-type ions was located at their N-termini (Scheme 1a) [5, 6]. This structure has often been cited [7-10].

Subsequently, they proposed a more general structure with various possible protonation sites (Scheme 1b) [11] and this has also been cited [4, 12]. This general structure has a peptide-like backbone frame with the ionizing proton at various positions, corresponding to a set of peptide ionic isomers. However, no experiments have elucidated which of these isomers is the preferred $y$-ion structure.

There is some disagreement in the literature regarding the structure of $b$-type ions. Two generic structures have been proposed: the acylium ion and the protonated oxazolone ion (Scheme 2) [11, 13].

The acylium ion structure-first proposed based on classical electron impact (EI) spectra [14] - is consistent with the loss of CO upon further activation to form $a$-type ions. However, this structure cannot explain the

Address reprint requests to Dr. James P. Reilly, Indiana University, Department of Chemistry, Chemistry Building, 800 E. Kirkwood Ave., Bloomington, IN 47405-4001. E-mail: reilly@indiana.edu relative deficiency of $b_{1}$ ions in CID spectra of peptides since $b_{1}$ acylium ions would be expected to be as stable as other $b$-type ions of this structure [15]. Furthermore, theoretical calculations indicate that the acylium ion structure is not thermodynamically stable given that the loss of CO to form a-type ions is exothermic [15]. The oxazolone structure was first proposed by Hunt and colleagues [13]. Extensive ab initio calculations have indicated the high stability of this structure [16]. Recent spectroscopic and theoretical evidences for oxazolone formation have been obtained by infrared multi-photon dissociation (IRMPD) spectroscopy in a Fourier transform ion cyclotron resonance (FT-ICR) mass spectrometer [17]. Because the acylium structure has a fixed charge located at the $\mathrm{C}$-terminus and the oxazolone ion has a mobile ionizing proton, determination of the charge location in $b$-type ions should therefore enable these two structures to be differentiated. Recently, a cyclic structure for $b$-type ions has been proposed by Paizs and coworkers based on sequence-scrambling fragmentation along with IR spectroscopy results in an FT-ICR mass spectrometer [18, 19]. However, this cyclic structure is produced in low abundance in beam-type CID performed in a quadrupole time-of-flight (Q-TOF) type mass spectrometer. This implies that the long timescale of sustained off-resonance irradiation (SORI) CID in the FT-ICR offers $b$ ions ample opportunity to form the cyclic structure [19]. To check whether the cyclic $b$ ions are produced in ion-trap CID, $b$ ions will be fragmented by both CID and photodissociation and evidence of sequence-scrambling fragmentation indicative of the cyclic structure will be sought.

Theoretical prediction of charge location in gasphase peptide ions is difficult because the protonation process is affected by various factors including amino acid proton affinity, peptide global conformation, and internal energy of ions [20]. Even though basic amino acids are normally regarded as the preferential proto- 
(a)<smiles>[R]C([NH3+])CC(=O)O</smiles>

(b)

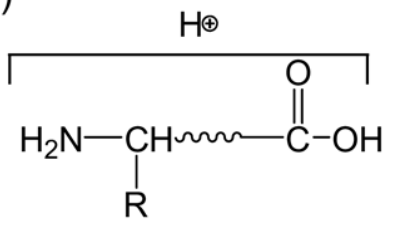

Scheme 1

nation sites [21], this prediction can often be wrong since the peptide conformation and intramolecular interactions might favor protonation at other sites [22, 23]. Experimental identification of protonation sites is also challenging because protons are really labile and appear to be easily mobilized upon activation [3]. Preservation of the proton location is therefore the key to a successful experiment.

Tandem mass spectrometry (MS/MS) techniques have been widely used to determine molecular structures based on the resulting fragment ions. To localize labile protons in peptide ions by MS/MS, the applied fragmentation method should not disturb the ionizing protons. Only a few fragmentation techniques meet this requirement. High-energy CID was first applied to probe protonation sites in peptide ions by Biemann and coworkers based on the observation that protons bound to a basic site in a peptide appeared to be sequestered during the high-energy fragmentation event [1, 24]. The interpretation was that high-energy CID is a charge-remote fragmentation that did not involve the ionizing proton(s). Electron capture dissociation (ECD) is another fragmentation technique introduced by McLafferty and colleagues to investigate multiply charged protein ions [25]. Through a dissociative electronic state, ECD does not mobilize protons during backbone fragmentation. Protonation sites in gaseous protein and peptide ions can therefore be identified by charge-state differences of consecutive $c$ - or $z$-fragment ions [26, 27]. However, this method cannot be applied to singly charged ions since ECD requires multiply charged precursors [25]. Furthermore, IRMPD spectroscopy in an FT-ICR mass spectrometer has been recently used to probe gaseous ion structures by matching experimental IR absorption spectra against theoretical spectra of calculated structures [17, 28]. Protonation sites can be assigned by different bending modes of $\mathrm{O}-\mathrm{H}$ and $\mathrm{N}-\mathrm{H}$ bonds in the IR spectra. However, this method requires high-level theoretical calculations on peptide conformation and energetics.

Although Biemann and associates demonstrated that high-energy CID was capable of localizing a peptide charge, their experiment involved a multiple-sector mass spectrometer to probe fragment ion structures [24, 29]. To perform $\mathrm{MS}^{n}$ experiments to elucidate molecular structures, ion trap mass spectrometers are now commonly used. However, CID in the ion trap is a lowenergy process that cannot be used to determine the charge location in peptide ions since collisional activa- tion mobilizes the ionizing proton before backbone cleavage [3, 30]. Moreover, 157-nm photodissociation can also be performed in an ion trap and considerable evidence suggests that it involves high-energy chargeremote peptide fragmentation [31-33]. When an arginine is present at the $\mathrm{N}$ - or C-terminus, it produces primarily $\mathrm{N}$ - or C-terminal fragments, respectively. On the other hand, for peptides without arginine, such as lysine-terminated peptides, it produces both $\mathrm{N}$ - and C-terminal fragments [32]. The simplest interpretation is that arginine sequesters the proton and the distribution of photofragment ions therefore reflects the initial charge location; when the charge is mobile, both $\mathrm{N}$ - and C-terminal fragments are produced. Therefore in 157-nm photofragmentation, observation of only Nterminal or C-terminal fragments indicates that the proton is sequestered on arginine; observation of both $\mathrm{N}$ - and C-terminal fragments would implicate a mobile proton that could be on any basic site. Based on these observations, we should be able to use 157-nm photodissociation to determine whether the proton on a singly charged peptide ion is indeed sequestered. In this work, we study not only intact peptide precursor ions, but, by exploiting the $\mathrm{MS}^{n}$ capability of the ion trap we also investigate charge location in peptide fragment ions generated from low-energy collision-induced dissociation.

\section{Experimental}

\section{Materials}

Peptides RFSWGAEGQ and RYLGYLE were purchased from Sigma (St. Louis, MO, USA). All other argininecontaining peptides were synthesized by Sigma Genosys (The Woodlands, TX, USA). Methanol (MeOH) was obtained from Mallinckrodt Baker, Inc. (Phillipsburg, NJ, USA). Acetonitrile (ACN) was purchased from EMD Chemicals, Inc. (Gibbstown, NJ, USA). Formic acid (FA) and acetic acid were obtained from Fluka Chemika $\mathrm{GmbH}$ (Buchs, Switzerland). Carboxypeptidase Y (CPY) was purchased from Worthington Biochemicals (Lakewood, NJ, USA) and Carboxypeptidase P (CPP) was bought from Takara Mirus Bio (Madison, WI, USA).

\section{Carboxypeptidase Digestion}

To shorten some of our prototype peptides, a carboxypeptidase digestion protocol was adapted from our previous work [34]. In brief, $100 \mu \mathrm{M}$ peptide

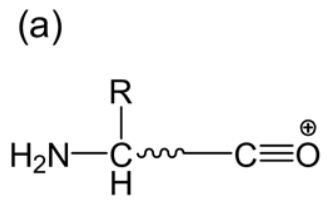

(b)

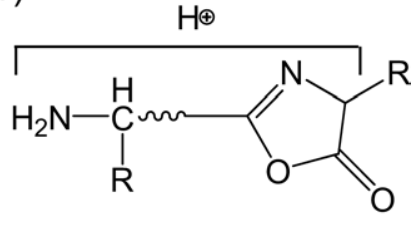

Scheme 2 
solutions were prepared in pyridine acetate buffer $(\mathrm{pH}$ 5.5). Five microliters of a solution containing $0.60 \mu \mathrm{g}$ of CPY and CPP or $1.20 \mu \mathrm{g}$ of CPY and CPP enzymes in pyridine acetate were then added. Digestions were performed at room temperature or at $37^{\circ} \mathrm{C}$. Aliquots were removed at several time points and acidified with $2 \%$ formic acid solution to quench the reaction. Peptide solutions were dried by a speed vac and stored in a freezer.

\section{Mass Spectrometry}

An LTQ linear ion trap mass spectrometer (ThermoFisher, San Jose, CA, USA) was used in this work for both CID and photodissociation. The photodissociation experiments were set up as previously reported [33, 35]. Briefly, peptides were ionized by electrospray. Singly charged precursor ions were isolated for photodissociation. An $\mathrm{F}_{2}$ laser (EX100HF-60, GAM laser, Orlando, FL, USA) generated light pulses that were introduced axially into the trap from its back side through an aperture $(1.7 \mathrm{~mm})$. It was triggered by the LTQ's activation signal, producing a 3-mJ, 10-ns pulse of 157-nm vacuum ultraviolet (VUV) light. After aperturing, only about $40 \mu \mathrm{J}$ of light actually entered the ion trap. Since the diameter of apertured light $(1.7 \mathrm{~mm})$ is much smaller than that of the ion cloud in the ion trap (about $4 \mathrm{~mm}$ ) [36], only a small portion of ions can be irradiated. Typically, one laser pulse is used for each photodissociation. All resulting fragment ions were scanned out to the dual detector as in its normal operating mode.

The ultraviolet light can photoionize some organic contaminants in the vacuum system, giving rise to reproducible photoionization products. This has been confirmed by the observation of such products without the introduction of analytes [33]. To remove these artifacts, background spectra were taken without injecting sample right after each experiment and subtracted from photodissociation spectra.

In this work, a combination of conventional lowenergy CID and photodissociation was used to probe CID fragment structures. In a typical experiment, peptides were prepared in $2 \mu \mathrm{M}$ solutions with $49.5 \% \mathrm{H}_{2} \mathrm{O}$, $49.5 \% \mathrm{ACN}$, and $1 \%$ of acetic acid and were directly infused into the electrospray ionization (ESI) source using a syringe pump. The flow rate was set at $3 \mu \mathrm{L} / \mathrm{min}$. Peptide ions were first dissociated by low-energy CID to produce $y$ - and $b$-type fragment ions. An activation time of $30 \mathrm{~ms}$ was set for the collisional activation. CID fragment ions were then isolated and subsequently photofragmented. The activation time in the $\mathrm{MS}^{3}$ stage was set at $30 \mathrm{~ms}$. Background subtraction was performed for every photodissociation spectrum.

\section{Results and Discussion}

\section{Generic y-Type Ions}

Most protein identification techniques involve fragmentation of tryptic peptides [8]. CID of these tryptic peptides generates many $y$-type ions along with a few $b$-type ions. Several doubly charged peptide ions with arginine at their C-termini were collisionally dissociated and the resulting $y$-type fragment ions were then photodissociated. Results for the $y_{6}$ fragment ion generated by CID of doubly charged peptide SAASINSR ions are displayed in Figure 1a. (The labeling indices for our $\mathrm{MS}^{3}$ spectra refer to the sequences of the CID fragment ions, not those of the precursor ions. For $\mathrm{N}$-terminal fragments formed in the photodissociation of C-terminal fragments, this obviously makes a difference.) Typical of photofragmentation spectra of peptides with arginine at their C-termini [31-33], dominant C-terminal fragment ions are observed, including a contiguous series of $x$-type ions extending from $x_{1}$ to $x_{4}$ along with some $y-, z-, v-$, and $w$-type ions. All of the fragments observed in this spectrum are consistent with retention of the ionizing proton at the C-terminal arginine after photoexcitation. This can be explained by two possible photofragmentation pathways. One is that the proton is sequestered on the arginine before photoexci-

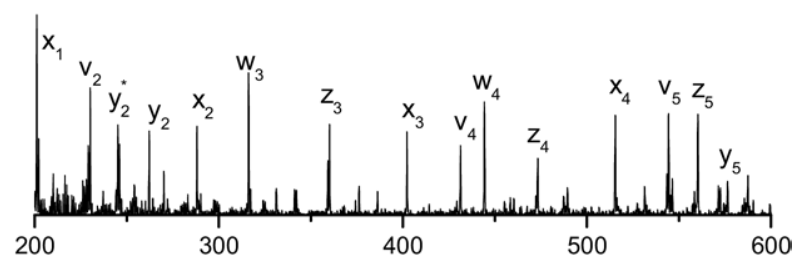

(a)

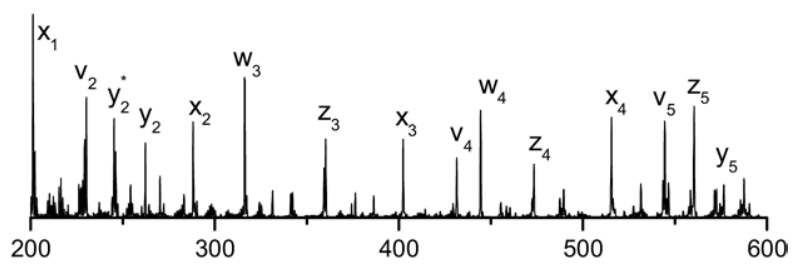

(b)

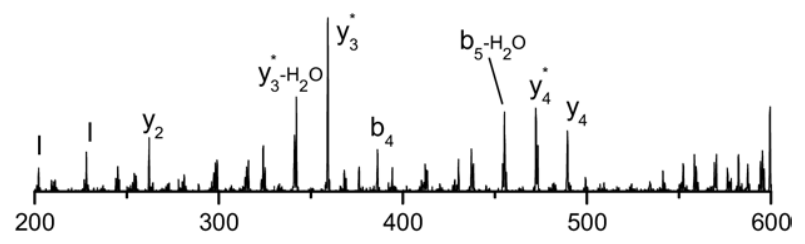

(c)

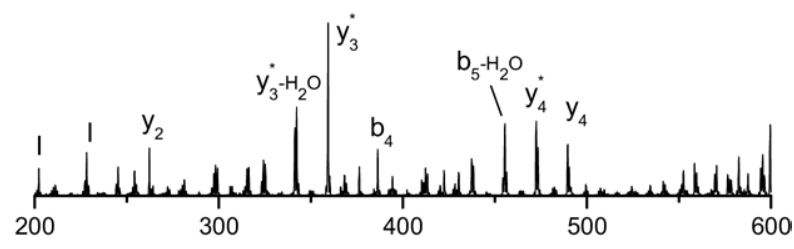

(d)

Figure 1. Photodissociation spectra of singly charged (a) $y_{6}$ ions produced by collisional dissociation of doubly charged SAASINSR ions and (b) peptide ASINSR ions. CID spectra of singly charged (c) $y_{6}$ ions produced by collisional dissociation of doubly charged SAASINSR ions and (d) peptide ASINSR ions. The asterisk (*) symbol denotes loss of ammonia in all figures. 
tation and it remains sequestered afterwards. The other is that the proton is not sequestered on arginine initially but it migrates to arginine after photoexcitation. We favor the first hypothesis. If a proton were mobilized by photoexcitation, it would not be trapped on arginine because the ion would be highly internally excited (the 157-nm photon energy is $7.9 \mathrm{eV}$ ) and the system will not be trapped in its lowest energy state unless energy is removed by collisional cooling with a third body. As demonstrated in our previous work, when the charge proton is not sequestered, photofragmentation produces both N- and C-terminal fragments [32]. Analogously, if the proton were mobilized during photofragmentation of $y$-type ions, both $\mathrm{N}$ - and C-terminal fragments would be expected. The fact that no Nterminal fragments have been observed strongly suggests that the proton is sequestered on arginine when a $y$-type ion is photofragmented.

To compare the structures of $y$-type ions with those of peptides of the same sequence, several truncated peptides were synthesized and then photodissociated. For example, Figure $1 \mathrm{~b}$ displays the photodissociation spectrum of peptide ASINSR that has the same sequence as that of the $y_{6}$ ion just discussed. It is virtually identical to that in Figure 1 a with the same $y^{-}, z_{-}^{-}, v^{-}$, and $w$-type ions. The spectral similarity confirms that the $y$-type fragment ion has the same structure (and in particular charge location) as that of the corresponding peptide.

Spectra obtained by collisionally dissociating $y_{6}$ fragment ions derived from SAASINSR and peptide ASINSR ions are shown in Figure $1 c$ and $d$, respectively. Both of these CID spectra contain $b$ - and $y$-type ions, internal fragments along with a number of uninterpretable components. Loss of $\mathrm{H}_{2} \mathrm{O}, \mathrm{NH}_{3}$, or both is commonly observed for the fragment ions, complicating the spectra. The detection of both $\mathrm{N}$ - and C-terminal fragments is typical of "thermal processes" in which activation energy is dispersed throughout the molecule and backbone bond cleavage is induced by a "mobile proton" $[3,4]$. The charge location cannot be discerned from these spectra presumably because there is no single fixed location after collisional activation.

\section{Effect of the Charge State of Peptide Ions on y- Type Ion Structures}

Enhanced charge-remote fragmentation at acidic amino acids has been observed for singly charged peptides but not their doubly charged counterparts [37]. Therefore, it is of interest to investigate whether the charge state of peptide precursor ions affects the structure of CIDgenerated fragment ions when cleavage occurs at these locations.

Both singly and doubly charged peptide EGVNDNEEGFFSAR ions were collisionally dissociated and the resulting $y_{7}$ ions were then photodissociated. Results are displayed in Figure 2a and b. In the accessible mass

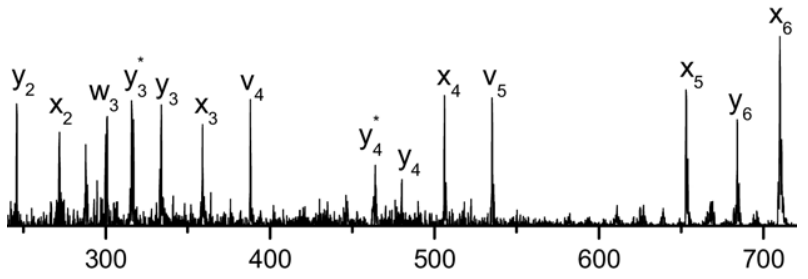

(a)

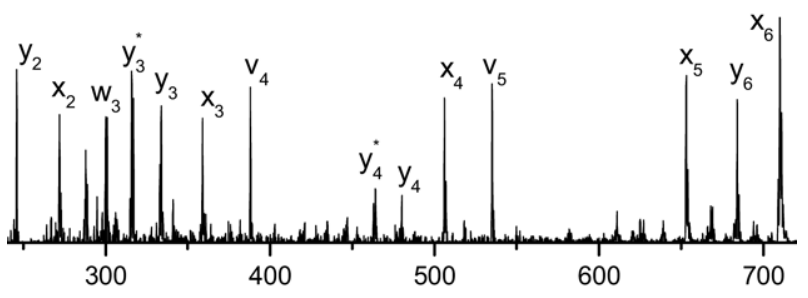

(b)

Figure 2. Photodissociation spectra of singly charged $y_{7}$ ions produced by collisional dissociation of (a) singly and (b) doubly charged EGVNDNEEGFFSAR ions.

region, contiguous $x$-type ions extending from $x_{2}$ to $x_{6}$ are observed along with a few $w_{-}, v_{-}$, and $y$-type ions. The ubiquity of C-terminal fragments implies that $y_{7}$ ions bear the ionizing charge at the $C$-terminal arginine. The data clearly demonstrate that $y_{7}$ ions generated from singly and doubly charged peptide ions have the same structure. Furthermore, other $y$ ions produced by the aspartic acid effect including $y_{6}, y_{9}$, and $y_{13}$ were also photodissociated. Results were similar to those obtained with $y_{7}$ ions. For the singly charged peptide ions, the generation of $y_{7}$ ions is induced by chargeremote fragmentation, in which the side-chain carboxylic acid of glutamic acid facilitates the backbone cleavage and the charge proton is sequestered on arginine $[37,38]$. Therefore, the resulting $y_{7}$ ions bear the charge on arginine. On the other hand, the doubly charged peptide ions have two ionizing protons: one is sequestered on arginine and the other is mobile [3,4]. Whether the peptide backbone fragmentation is induced by the side-chain carboxylic acid or the mobile proton, the proton on arginine remains sequestered during the formation of $y$-type ions. Therefore, for peptides containing acidic amino acids, it is reasonable that $y$-type fragments have the same structure even though they might have arisen from different precursor ions that fragment in different ways.

Other $y_{7}$ ions generated from collisional dissociation of singly charged and doubly charged FSWGAEGQR were photodissociated and their spectra are displayed in Figure $3 a$ and b. Again the spectra are essentially identical and contain a contiguous series of $x$ ions along with a few $v_{-}, w_{-}$, and $y$-type ions. All of these fragments correspond to charge retention on the C-terminal arginine. It confirms that the $y$-type fragment ion structure is not affected by the charge state of the precursor ion. It is widely accepted that low-energy CID is facilitated by a mobile proton [1-4]. Based on this, the collisional 


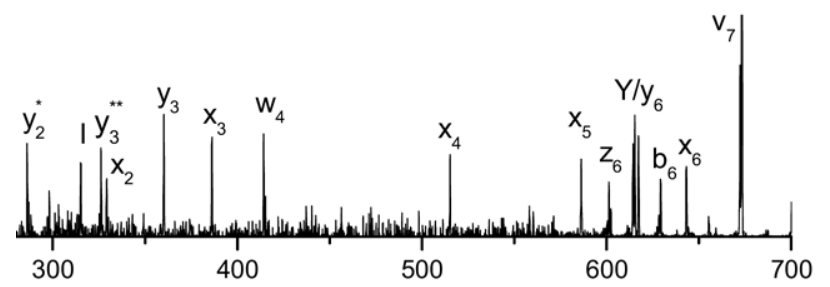

(a)

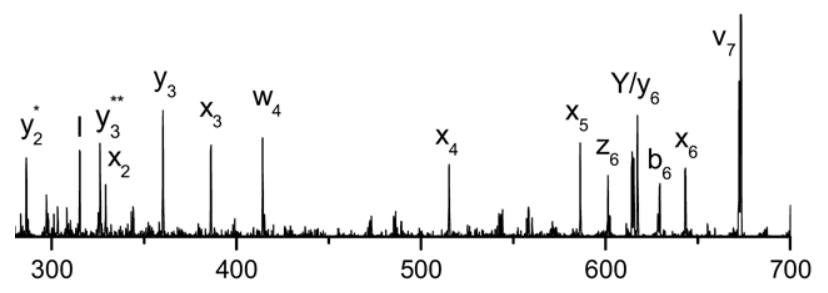

(b)

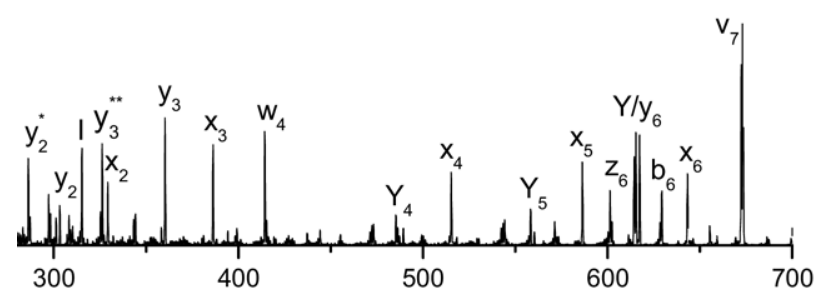

(c)

Figure 3. Photodissociation spectra of singly charged $y_{7}$ ions produced by collisional dissociation of (a) singly and (b) doubly charged FSWGAEGQR ions. (c) Photodissociation spectrum of singly charged WGAEGQR. The double asterisks $\left({ }^{* *}\right)$ denotes loss of two ammonias.

dissociation of singly charged peptide should produce $y$-type fragment ions with the charge at the $\mathrm{N}$-terminal amine. However, we have shown that at the time of photofragmentation, the ionizing proton in $y$-type fragment ions resides at the C-terminal arginine. Presumably this results from a proton migration process although it is still not obvious whether this occurs right after the collisional dissociation or during $y$-ion isolation.

Peptide WGAEGQR has the same sequence as that of the $y_{7}$ fragment from the previous example. The photodissociation spectrum of the singly charged peptide in Figure $3 c$ is essentially identical to spectra in Figure 3a and $b$, confirming that the peptide and $y$-type fragment ions have the same structure.

It is noteworthy that in all of the Figure 3 spectra, an $\mathrm{N}$-terminal fragment, $b_{6}$, appears. This is unexpected for a peptide with a C-terminal arginine and it may result from enhanced backbone amide bond cleavage involving the ionizing proton on the adjacent C-terminal arginine.

Photofragmentation of more than a dozen $y$-type ions yielded results similar to those of the previous three examples. The ionizing proton in $y$-type ions is located on the arginine and their structures are like those of peptides of the same sequence. This is analogous to $y$-type ions generated from charge-tagged pep- tides, in which the charge is always sequestered on a C-terminal charge tag [39].

\section{y-Type Ions with More Than One Protonation Site}

Both singly and doubly charged peptide AAPAAAR were collisionally dissociated and the resulting $y_{5}$ ions were then photodissociated. Results for photodissociation are displayed in Figure 4a and b. It is obvious that the two photodissociation spectra are essentially identical, each consisting of a series of C-terminal fragments including $x-, v-$, and $y$-type ions and several $N$-terminal fragments including $a$ - and $b$-type ions. As shown before, the $x$ - and $v$-type ions result from retention of the charge on arginine. However, the detection of multiple $\mathrm{N}$-terminal fragments including $a$ - and $b$-type ions indicates that the charge proton in some $y_{5}$ ions is not sequestered on arginine. Proline has a tertiary backbone amine that is about $32 \mathrm{~kJ} \mathrm{~mol}^{-1}$ more basic than that of other amino acids, making it an attractive protonation site $[21,40]$. However, it is noteworthy that most of the observed $\mathrm{N}$-terminal fragments are $b$-type ions that are not abundantly produced during photodissociation of peptides with an N-terminal arginine. This indicates that the ionizing proton on the proline is not sequestered as it is on the arginine. It is still not obvious whether the proton migration process occurs before CID or only after the collisional activation. Previous theoretical calculations showed that proton

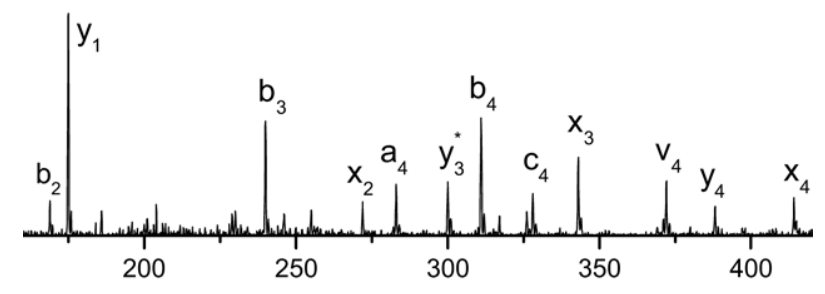

(a)

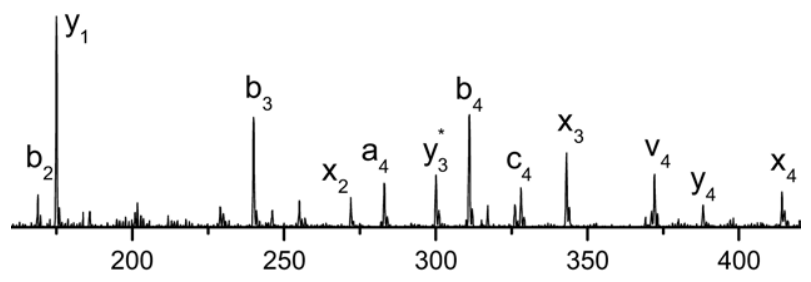

(b)

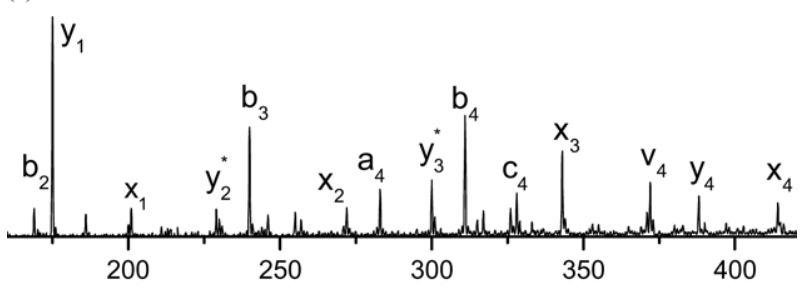

(c)

Figure 4. Photodissociation spectra of singly charged $y_{5}$ ions produced by collisional dissociation of (a) singly and (b) doubly charged peptide AAPAAAR ions. (c) Photodissociation spectrum of singly charged PAAAR ions. 
migration in the gas phase requires relatively little energy (about $23 \mathrm{kcal} \mathrm{mol}^{-1}$ ) $[25,40]$. Therefore the ionizing proton could migrate in an ion trap since this energy barrier can be overcome during regular ion trap operation.

The photodissociation spectrum of singly charged PAAAR peptide ions is displayed in Figure 4c. It is essentially identical to that of the $y_{5}$ ions and contains both C-terminal and N-terminal fragments. Williams and colleagues reported that proline and some other non-basic residues can also be protonated during ESI of peptides and proteins [22, 41]. Our fragmentation results indicate both proline and arginine residues are protonated during ESI, which is consistent with the previous observations.

The position of the ionizing proton in $y$-type ions terminated with basic amino acids, including histidine and lysine, was also investigated. $y_{6}$ ions generated from both GDHGGGGR and GDKGGGGR were photodissociated. Similar to Figure 4, spectra are composed of both $\mathrm{N}$ - and C-terminal fragment, indicating that the ionizing proton is located on either arginine or the other basic residue. These results are analogous to those obtained from proline-terminated $y$-type ions.

\section{Generic b-Type Ions}

CID of peptides with an N-terminal arginine generally produces abundant $b$-type ions. In an attempt to learn about the charge location in these ions, several peptide ions with $\mathrm{N}$-terminal arginine were collisionally dissociated and the resulting $b$-type ions were photodissociated. Data for the $b_{8}$ fragment ion generated by CID of doubly charged RFSWGAEGQ are displayed in Figure 5a. A contiguous series of $a$-type ions, extending from $a_{2}$ to $a_{8}$, and a few $d$ - and $b$-type ions are observed. No C-terminal fragments are present. All of these fragments correspond to retention of the charge proton at the $\mathrm{N}$-terminus. The intense $a_{8}$ ions associated with a loss of $\mathrm{CO}$ from $b_{8}$ ions are typical for $b$-type ion fragmentation. The CID spectrum of the $b_{8}$ ion is shown in Figure $5 \mathrm{~b}$. It contains a few large $a$ - and $b$-type ions in the high-mass region, although below 600 Da no strong peaks are present. The abundant production of $b_{7}$ is consistent with the previous report by Harrison and colleagues [42]. Their work suggested that the cleavage of the terminal residue is facilitated by the nucleophilic attack of the next-nearest carbonyl on the terminal oxazolone ring and $b_{n}$ ions therefore preferentially fragment to form next-lower $b_{n-1}$ ions. The lack of small $b_{n}$ in our CID spectra is presumably a consequence of our use of an ion trap that resonantly excites only precursor ions and therefore favors energetically preferential fragmentation pathways. Harrison's mechanism involves a charged oxazolone ring structure that is formed in low-energy collisional excitation during which charge mobilization occurs. However, our detection of only N-terminal fragments in the 157-nm photodissociation experiment indicates that the charge pro-

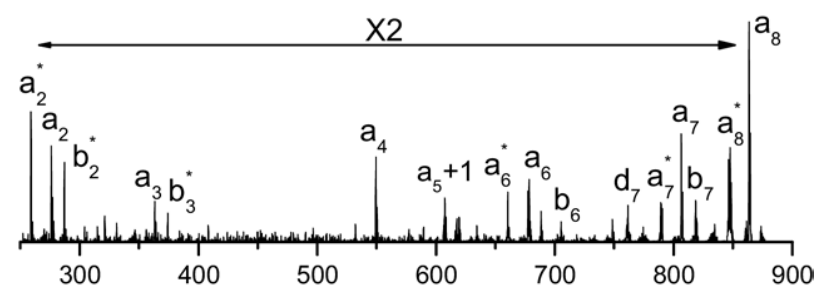

(a)

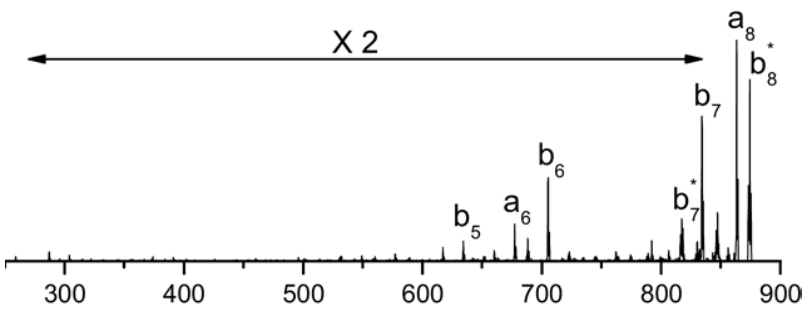

(b)

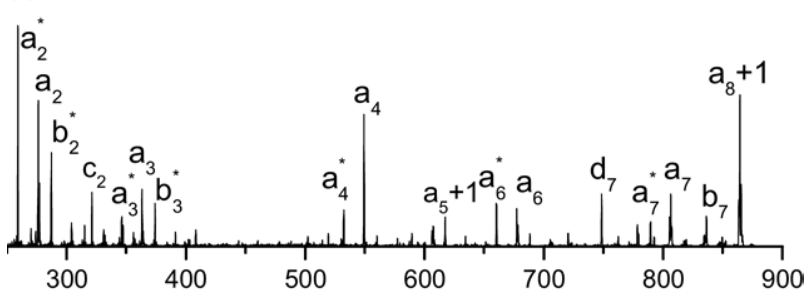

c)

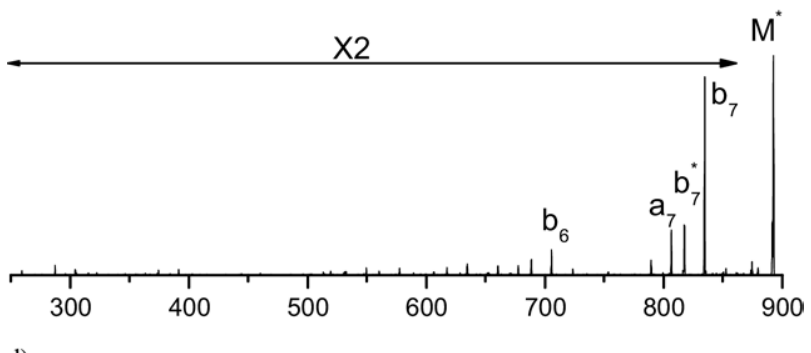

d)

Figure 5. (a) Photodissociation and (b) CID spectra of singly charged $b_{8}$ ions produced by collisional dissociation of doubly charged RFSWGAEGQ ions. (c) Photodissociation and (d) CID spectra of singly charged RFSWGAEG.

ton is sequestered on arginine and it is not mobilized by photoexcitation. In addition, photodissociation evidently introduces more energy, enabling many bonds to be broken.

For a comparison with the preceding results, peptide RFSWGAEG was made by using carboxypeptidase to cleave the $C$-terminal glutamine from peptide RFSWGAEGQ [34]. Its photodissociation and CID spectra are displayed in Figure $5 c$ and d. The photodissociation spectra of the peptide and the $b_{8}$ ion are very similar in the low-mass region. However, they vary somewhat at high mass. For the peptide, both $a_{8}+1$ and $a_{8}$ ions are observed. The $a_{8}+1$ ion corresponds to radical cleavage of a carboxylic group from either the C-terminus or the glutamic acid side chain and the $a_{8}$ ion is formed via loss of a hydrogen atom from the $a_{8}+1$ radical ion [35, 43]. However, in the photodissociation 
spectrum of the $b_{8}$ ion, no $a_{8}+1$ ions are observed. Instead, $a_{8}$ ions are formed via loss of CO. The CID spectrum of the peptide is also similar to the CID spectrum of the $b_{8}$ ion, except that no $a_{8}$ ions are observed from the peptide. Even though it is well known that a peptide and a $b$-type ion of the same sequence have different structures, our photodissociation results indicate that an N-terminal arginine sequesters an ionizing proton in both cases.

It is noteworthy that our photodissociation and CID results show no evidence of sequence-scrambling fragments. It suggests that the ion trap CID of peptides does not primarily produce cyclic $b$-type ions, although they were abundantly produced in an FT-ICR mass spectrometer [19]. In the same work, Paizs and colleagues also found that the cyclic $b$-type ions were not abundantly produced by beam-type CID in a Q-TOF type mass spectrometer and they attributed this to the short dissociation timescale in the quadrupole instrument compared to that in an FT-ICR [19]. Similar to quadrupole beam-type CID, the time period during which collisional activation occurs in an ion trap CID process (30 ms) is also much shorter than SORI CID in the FT-ICR (a few seconds), which might disfavor the formation of the cyclic $b$-type ions. In addition, $b$-type ions investigated in this work contain an N-terminal arginine that tends to sequester the charge proton, which may also disfavor the cyclization process of $b$-type ions.

$b_{5}$ ions generated from RLEQFG were photodissociated and the results are displayed in Figure 6a. A contiguous series of $a$-type ions extending from $a_{2}$ to $a_{8}$, and a few $d$ - and $b$-type ions are observed. The distribution of fragment ions confirms the retention of charge at the N-terminus. In addition, enhanced side-chain losses from $\mathrm{E}$ and $\mathrm{F}$ residues have been observed. Similar side-chain fragmentation has been also ob-

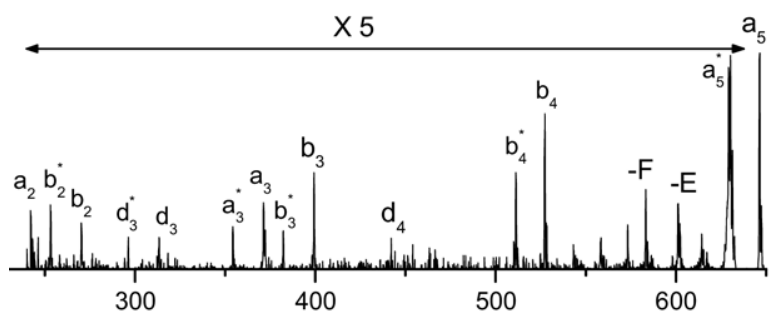

(a)

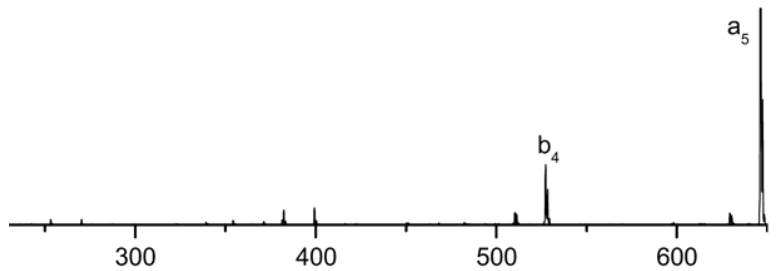

(b)

Figure 6. (a) Photodissociation and (b) CID spectra of singly charged $b_{5}$ ions produced by collisional dissociation of doubly charged RLEQFG ions. served in UV dissociation experiments using either 193or 266-nm light $[44,45]$. This has been attributed to the strong absorption by the carboxylic and aromatic groups on the amino acid side chain. Excitation of these groups leads to a fast dissociation of side-chain $\mathrm{C}_{\alpha}-\mathrm{C}_{\beta}$ bonds. Our 157-nm photofragmentation displays this same effect. The CID spectrum of the $b_{5}$ ion is shown in Figure $6 b$. Similar to Figure $5 b$, the tendency is to form only high-mass fragment ions. Results are consistent with charge retention on the N-terminal arginine.

Photodissociation of more than a dozen $b$-type ions with an N-terminal arginine yielded results similar to spectra in Figures 5 and 6. The ionizing proton in the $b$-type ions is at the $\mathrm{N}$-terminal arginine residue. We observed no evidence for an acylium structure that has a fixed charge on its C-terminus. Even though the initially proposed oxazolone structure had the charge on the ring [13], the general oxazolone structure drawn by Biemann has an ionizing proton that can be found anywhere in the ion (Scheme 2) [11]. Our results indicate that the ionizing proton is sequestered on the $\mathrm{N}$ terminal arginine.

\section{Conclusion}

Photodissociation of CID-produced fragment ions reveals rich structural information. $y$-type ions have the structure of peptides of the same sequence with the ionizing proton located at the most basic sites(s). When more than one basic residue is present, ionic isomers with different charge positions can be formed. Photodissociation of generic $b$-type ions having an $\mathrm{N}$-terminal arginine reveals that the arginine sequesters the ionizing proton. No evidence for an acylium structure is observed.

\section{Acknowledgments}

This work has been supported by National Science Foundation Grants CHE0518234 and CHE0431991 and the Indiana METACyt Initiative of Indiana University, founded in part through a major grant from the Lilly Endowment, Inc.

\section{References}

1. Johnson, R. S.; Martin, S. A.; Biemann, K. Collision-Induced Fragmentation of $(\mathrm{M}+\mathrm{H})^{+}$Ions of Peptides. Side Chain Specific Sequence Ions. Int. J. Mass Spectrom. Ion Process. 1988, 86, 137-154.

2. Burlet, O.; Orkiszewski, R. S.; Ballard, K. D.; Gaskell, S. J. Charge Promotion of Low-Energy Fragmentation of Peptide Ions. Rapid Commun. Mass Spectrom. 1992, 6, 658-662.

3. Dongre, A. R.; Jones, J. L.; Somogyi, A.; Wysocki, V. H. Influence of Peptide Composition, Gas-Phase Basicity, and Chemical Modification on Fragmentation Efficiency: Evidence for the Mobile Proton Model. J. Am. Chem. Soc. 1996, 118, 8365-8374.

4. Wysocki, V. H.; Tsaprailis, G.; Simth, L. L.; Breci, L. A. Mobile and Localized Protons: A Framework for Understanding Peptide Dissociation. J. Mass Spectrom. 2000, 35, 1399-1406.

5. Roepstorff, P.; Fohlman, J. Proposal for a Common Nomenclature for Sequence Ions in Mass Spectra of Peptides. Biomed. Mass Spectrom. 1984, 11,601 .

6. Biemann, K. Contributions of Mass-Spectrometry to Peptide and Protein-Structure. Biomed. Environ. Mass Spectrom. 1988, 16, 99-111.

7. Vaisar, T.; Urban, J. Gas-Phase Fragmentation of Protonated Monon-NMethylated Peptides. Analogy with Solution-Phase Acid-Catalyzed Hydrolysis. J. Mass Spectrom. 1998, 33, 505-524. 
8. Aebersold, R.; Goodlett, D. R. Mass Spectrometry in Proteomics. Chem. Rev. 2001, 101, 269-295.

9. Paizs, B.; Suhai, S. Towards Understanding the Tandem Mass Spectra of Protonated Oligopeptides. 1: Mechanims of Amide Bond Cleavage. J. Am. Soc. Mass Spectrom. 2004, 15, 103-113.

10. Paizs, B.; Suhai, S. Fragmentation Pathways of Protonated Peptides. Mass Spectrom. Rev. 2005, 24, 508-548.

11. Biemann, K. Nomenclature for Peptide Fragment Ions (Positive Ions). Methods Enzymol. 1990, 193, 886-887.

12. Smith, L. L.; Herrmann, K. A.; Wysocki, V. H. Investigation of Gas Phase Ion Structure for Proline-Containing $b_{2}$ Ion. J. Am. Soc. Mass Spectrom. 2005, 17, 20-28.

13. Arnott, D.; Kottmeir, D.; Yates, N.; Shabanowitz, J.; Hunt, D. F. Proceedings of the 42nd ASMS Conference on Mass Spectrometry, Chicago, IL, June, 1994; p. 470.

14. McLafferty, F. W.; Tureček, F. Interpretation of Mass Spectra, 4th ed.; University Science: Sausalito, CA, 1993.

15. van Dongen, W. D.; Heerma, W.; Haverkamp, J. The b1-Fragment Ion from Protonated Glycine Is an Electrostatically-Bound Ion/Molecule Complex of $\mathrm{CH}_{2}=\mathrm{NH}_{2}{ }^{+}$and CO. Rapid Commun. Mass Spectrom. 1996, 10, 1237-1239.

16. Paizs, B.; Lendvay, G.; Vekey, K.; Suhai, S. Formation of $b_{2}{ }^{+}$Ions from Protonated Peptides: An Ab Initio Study. Rapid Commun. Mass Spectrom. 1999, 13, 525-533.

17. Polfer, N. C.; Oomens, J.; Suhai, S.; Paizs, B. Spectroscopic and Theoretical Evidence of Oxazolone Ring Formation in Collision-Induced Dissociation of Peptides. J. Am. Chem. Soc. 2005, 127, 17154-17155.

18. Harrison, A. G.; Young, A. B.; Bleiholder, C.; Suhai, S.; Paizs, B. Scrambling of Sequence Information in Collision-Induced Dissociation of Peptides. J. Am. Chem. Soc. 2006, 128, 10364-10365.

19. Polfer, N. C.; Oomens, J.; Suhai, S.; Paizs, B. Infrared Spectroscopy and Theoretical Studies on Gas-Phase Protonated Leu-Enkephalin and Its Fragments: Direct Experimental Evidence for the Mobile Proton. J. Am. Chem. Soc. 2007, 129, 5887-5897.

20. Hoaglund-Hyzer, C. S.; Counterman, A. E.; Clemmer, D. E. Anhydrous Protein Ions. Chem. Rev. 1999, 99, 3037-3079.

21. Harrison, A. G. The Gas-Phase Basicities and Proton Affinities of Amino Acids and Peptides. Mass Spectrom. Rev. 1997, 16, 201-217.

22. Schnier, P. D.; Price, W. D.; Jockusch, R. A.; Williams, E. R. Blackbody Infrared Radiative Dissociation of Bradykinin and Its Analogues: Energetics, Dynamics, and Evidence for Salt-Bridge Structures in the Gas Phase. J. Am. Chem. Soc. 1996, 118, 7178-7189.

23. Wyttenbach, T.; Paizs, B.; Barran, P.; Breci, L.; Liu, D.; Suhai, S.; Wysocki, V. H.; Bowers, M. T. The Effect of the Initial Water of Hydration on the Energetics, Structures and H/D Exchange Mechanism of a Family of Pentapeptides: An Experimental and Theoretical Study. J. Am. Chem. Soc. 2003, 125, 13768-13775.

24. Downard, K. M.; Biemann, K. Charging Behavior of Highly Basic Peptides During Electrospray Ionization. A Predilection for Protons. Int. J. Mass Spectrom. Ion Process. 1995, 148, 191-202.

25. Zubarev, R. A.; Kelleher, N. L.; McLafferty, F. W. Electron Capture Dissociation of Multiply Charged Protein Cations-A Nonergodic Process. J. Am. Chem. Soc. 1998, 120, 3265-3266.

26. Breuker, K.; Oh, H.; Horn, D. M.; Cerda, B. A.; McLafferty, F. W. Detailed Unfolding and Folding of Gaseous Ubiquitin Ions Characterized by Electron Capture Dissociation. J. Am. Chem. Soc. 2002, 124, 6407-6420.

27. Kjeldsen, F.; Savitski, M. M.; Adams, C. M.; Zubarev, R. A. Determination of the Location of Positive Charges in Gas-Phase Polypeptide Polycations by Tandem Mass Spectrometry. Int. J. Mass Spectrom. 2006, 252, 204-212.
28. Polfer, N. C.; Paizs, B.; Snoek, L. C.; Compagnon, I.; Suhai, S.; Meijer, G.; von Helden, G.; Oomens, J. Infrared Fingerprint Spectroscopy and Theoretical Studies of Potassium Ion Tagged Amino Acids and Peptides in the Gas Phase. J. Am. Chem. Soc. 2005, 127, 8571-8579.

29. Sullards, M. C.; Reiter, J. A. Primary and Secondary Locations of Charge Sites in Angiotensin II $(\mathrm{M}+2 \mathrm{H})^{2+}$ Ions Formed by Electrospray Ionization. J. Am. Soc. Mass Spectrom. 2000, 11, 40-53.

30. Tang, X.; Thibault, P.; Boyd, R. K. Fragmentation Reactions of MultiplyProtonated and Implications for Sequencing by Tandem MassSpectrometry with Low-Energy Collision-Induced Dissociation. Anal. Chem. 1993, 65, 2824-2834.

31. Thompson, M. S.; Cui, W.; Reilly, J. P. Fragmentation of Singly Charged Peptide Ions by Photodissociation at Lambda $=157 \mathrm{~nm}$. Angew. Chem. Int. Ed. 2004, 43, 4791-4794.

32. Cui, W.; Thompson, M. S.; Reilly, J. P. Pathways of Peptide ion Fragmentation Induced by Vacuum Ultraviolet Light. J. Am. Soc. Mass Spectrom. 2005, 16, 1384-1398.

33. Kim, T. Y.; Thompson, M. S.; Reilly, J. P. Peptide Photodissociation at $157 \mathrm{~nm}$ in a Linear Ion Trap Mass Spectrometer. Rapid Commun. Mass Spectrom. 2005, 19, 1657-1665.

34. Running, W. E.; Ravipaty, S.; Karty, J. A.; Reilly, J. P. A Top-Down/ Bottom-Up Study of the Ribosomal Proteins of Caulobacter crescentus. J. Prot. Res. 2007, 6, 337-347.

35. Zhang, L.; Cui, W.; Thompson, M. S.; Reilly, J. P. Structures of a-Type Ions Formed in the $157 \mathrm{~nm}$ Photodissociation of Singly-Charged Peptide Ions. J. Am. Soc. Mass Spectrom. 2006, 17, 1315-1321.

36. Blake, T. A.; Zheng, O.; Wiseman, J. M.; Takats, Z. Guymon, A. J. Kothari, S.; Cooks, R. G. Preparative Linear Ion Trap Mass Spectrometer for Separation and Collection of Purified Proteins and Peptides in Arrays Using Ion Soft Landing. Anal. Chem. 2004, 76, 6293-6305.

37. Tsaprailis, G.; Nair, H.; Somogyi, A.; Wysocki, V. H.; Zhong, W.; Futrell, J. H.; Summerfield, S. G.; Gaskell, S. J. Influence of Secondary Structure on the Fragmentation of Protonated Peptides. J. Am. Chem. Soc. 1999, 121, 5142-5154

38. Tsaprailis, G.; Somogyi, A.; Nikolaev, E. N.; Wysocki, V. H. Refining the Model for Selective Cleavage at Acidic Residues in Arginine-Containing Protonated Peptides. Int. J. Mass Spectrom. 2000, 195/196, 467-479.

39. Watson, J. T.; Wagner, D. S.; Chang, Y. S.; Strahler, J. R.; Hanash, S. M.; Gage, D. A. Characterization of the Ethyl-Triphenylphosphonium Derivative of Model Peptides by Fast Atom Bombardment CollisionallyActivated Dissociation Tandem Mass Spectrometry Using B/E Linked Scans. Int. I. Mass Spectrom. Ion Process. 1991, 111, 191-209.

40. Schwartz, B. L.; Bursey, M. M. Some Proline Substituent Effects in the Tandem Mass Spectrum of Protonated Pentaalanine. Biol. Mass Spectrom. 1992, 21, 92-96.

41. Schnier, P. D.; Gross, D. S.; Williams, E. R. On the Maximum Charge State and Proton Transfer Reactivity of Peptide and Protein Ions Formed by Electrospray Ionization. J. Am. Soc. Mass Spectrom. 1995, 6, 1086-1097.

42. Yalcin, T.; Csizmadia, I. G.; Peterson, M. R.; Harrison, A. G. The Structure and Fragmentation of $B_{n}(n \geq 3)$ Ions in Peptide Spectra. J. Am. Soc. Mass Spectrom. 1996, 7, 233-242.

43. Kjeldsen, F.; Silivra, O. A.; Zubarev, R. A. Zwitterionic States in Gas-Phase Polypeptide Ion Revealed by $157-\mathrm{nm}$ Ultra-Violet Photodissociation. Chem. Eur. J. 2006, 12, 7920-7928.

44. Guan, Z.; Kelleher, N. L.; O'Connor, P. B.; Aaserud, D. J.; Little, D. P.; McLafferty, F. W. 193 nm Photodissociation of Larger Multiply-charged Biomolecules. Int. J. Mass Spectrom. Ion Process. 1996, 158, 357-364.

45. Tabarin, T.; Antoine, R.; Broyer, M.; Dugourd, P. Specific Photodissociation of Peptides with Multi-stage Mass Spectrometry. Rapid Commun Mass Spectrom. 2005, 19, 2883-2892. 